\title{
Propagating waves transverse to the magnetic field in a solar prominence
}

\author{
Therese Kucera $^{1}$, Brigitte Schmieder ${ }^{2}$, Kalman Knizhnik ${ }^{3,1}$, \\ Arturo Lopez-Ariste ${ }^{4}$, Manuel Luna ${ }^{5}$ and David Toot $^{6}$ \\ ${ }^{1}$ NASA/GSFC, Greenbelt, MD, USA, email: therese.a.kucera@nasa.gov \\ ${ }^{2}$ Observatoire de Paris, LESIA, Meudon, 92195, France, \\ email: brigitte.schmieder@obspm.fr \\ ${ }^{3}$ Johns Hopkins University, Baltimore, MD USA ${ }^{4}$ THEMIS, CNRS , E38205 LaLaguna, Spain \\ ${ }^{5}$ Instituto de Astrofsica de Canarias, E38205 LaLaguna, Spain \\ ${ }^{6}$ Alfred University, Alfred, NY, USA
}

\begin{abstract}
We have observed a quiescent prominence with the Hinode Solar Optical Telescope (SOT) (Ca II and $\mathrm{H} \alpha$ lines), Sacramento Peak Dunn Solar Telescope using the Universal Birefringent Filter (DST/UBF, in $\mathrm{H} \alpha, \mathrm{H} \beta$ and Sodium-D lines), THEMIS (Télescope Héliographique pour 1 Etude du Magnétisme et des Instabilités Solaires/MTR (Multi Raies) spectromagnetograph $\left(\mathrm{He}_{3}\right)$, and the Solar Dynamics Observatory Atmospheric Imaging Assembly (SDO/AIA) in EUV over a 4 hour period on 2012 October 10. The small fields of view of the SOT, DST, and MTR are centered on a large prominence footpoint extending towards the surface. This feature appears in the larger field of view of the AIA/304 $\AA$ filtergram as a large, quasi-vertical pillar with loops on each side. The THEMIS/MTR data indicate that the magnetic field in the pillar is essentially horizontal and the observations in the optical domain show a large number of horizontally aligned features in the pillar. The data are consistent with a model of cool prominence plasma trapped in the dips of horizontal field lines. The SOT and DST data show what appear to be moving wave pulses. These pulses, which include a Doppler signature, move vertically, perpendicular to the field direction, along quasi-vertical columns of horizontal threads in the pillar. The pulses have a velocity of propagation of about $10 \mathrm{~km} / \mathrm{s}$, a wavelength about $2000 \mathrm{~km}$ in the plane of the sky, and a period about $280 \mathrm{sec}$. We interpret these waves in terms of fast magnetosonic waves.
\end{abstract}

Keywords. Sun: prominences, magnetic field, oscillations

\section{Prominence observed with Hinode/SOT, DST and THEMIS}

The prominence is observed by SDO/AIA (Fig. 1 left panel) in $304 \AA$. The fields of view of Hinode/SOT in Ca II H (Fig. 1 middle panel) and of the THEMIS vector magnetograph working in $\mathrm{He}_{3} \mathrm{D}_{3}$ are pointed at the central footpoint of the prominence. The raw data of the THEMIS/MTR mode was reduced with the DeepStokes procedure (Lopez et al. 2009). The Stokes profiles are fed to an inversion code based on Principal Component Analysis (Casini et al. 2003). The database used contains 90,000 profiles. The inferred magnetic field strength is in the range 5-10 Gauss, with an uncertainty of 2 Gauss, the inclination is estimated to be $90^{\circ} \pm 10^{\circ}$, and the azimuth is about $110^{\circ}$ from the plane defined by the line of sight and local vertical. Thus we conclude that the magnetic field in the locations of the cool prominence material is horizontal and about $30^{\circ}$ from the plane of the sky (Schmieder et al. 2013). 

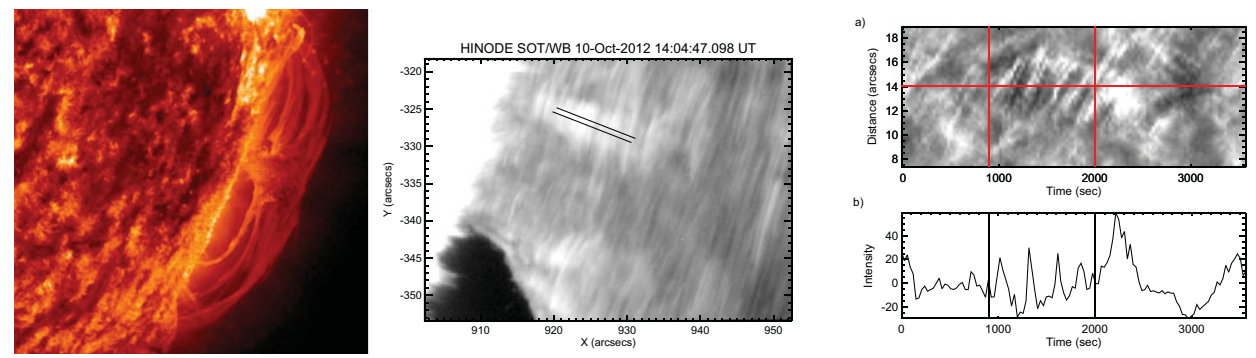

Figure 1. Prominence of 2012 October 10, observed by SDO/AIA in $304 \AA$ (left), by Hinode/SOT in Ca II H (middle), and an intensity map as a function of time along a long narrow box through the brightest column (top right), and a cut along a horizontal line in the time vs. distance plot (bottom right).

\section{Oscillations: Observations and Theory}

We integrated the intensity across a $0.55^{\prime \prime}(5$-pixel) wide area positioned across the oscillating region (Fig. 1 middle panel). The distance between each intensity peak (Fig. 1 right panels) is approximately $2000 \mathrm{~km}$. The slopes of the intensity peaks in these plots of time vs. distance correspond to the upwards velocity of the moving features, approximately $10 \mathrm{~km} / \mathrm{s}$. Fourier and wavelet analyses give a wave period of $277 \pm 50 \mathrm{sec}$, which appears to be approximately constant. We have modeled the oscillations as a fast magnetosonic wave in an uniform atmosphere. The driver oscillates three times with a period of $300 \mathrm{sec}$, similar to the observed one. The prominence-slab has a total width of 10 Mm. The magnetic field is taken to be uniform and horizontal ( $B=7.5$ Gauss). It is a similar equilibrium configuration to that of Joarder and Roberts (1992). The model prominence electron density $\left(10^{11} \mathrm{~cm}^{-3}\right)$ is 200 times larger than that of the surrounding corona and also on the high end of measured prominence densities (Labrosse et al. 2010). The temperature of the plasma is $8000 \mathrm{~K}$. We impose pressure perturbations.

\section{Conclusion}

The field strength in the bright columns is 5-10 Gauss and the magnetic field vector is mainly horizontal, confirming previous results. Propagating waves with periods around $300 \mathrm{sec}$ are detected. The Doppler shifts measured in $\mathrm{H} \beta$ show maxima during the passage of the wave. The driver of these waves is unknown. A simulation of a train of waves traveling upward producing disturbances of the gas pressure, the velocity and the magnetic field was performed. The wave front is almost planar in the prominence, oscillating vertically and horizontally in the corona with larger perturbations in the cool plasma than in the corona. The phase speed is the fast magnetosonic velocity of a uniform medium. This simulation demonstrates that the propagating waves are consistent with a fast magnetosonic wave confined to the prominence. However, the phase speed is much larger than the one observed. This could be due to projection effects.

\section{References}

Casini R., Lopez Ariste A., Tomczyk S. \& Lites B. W. 2003, ApJ, 598, L67

Labrosse N., Heinzel P., Vial J.-C., Kucera T., Parenti S., Gunár S., Schmieder, B. \& Kilper G. 2010, Space Sci. Rev., 151, 243

Lopez-Ariste A., Asensio Ramos A., Manso Sainz R., et al. 2009, ApJ, 501, 729

Joarder P. S. \& Roberts B. 1992, A\& A 261, 625

Schmieder B., Kucera T., Knizhnik K., Lopez-Ariste A., Luna M. \& Toot D. 2013, ApJ, 777, 108 